\title{
Exploration of Various Network Coding Schemes to Improve Performance in Wireless Multihop Networks
}

\author{
Sunil Bajeja \\ Asst. Prof. - FCA \\ Marwadi Edu. Fou. Grp of Inst. \\ Rajkot
}

\author{
Atul Gosai, PhD \\ Associate Prof. - CSD \\ Saurashtra University \\ Rajkot
}

\begin{abstract}
Network coding is a collection of techniques in which a node is allowed to combine, encode and send one or more packets as a single encoded packet instead of sending individually. It improves throughput, packet delivery ratio and efficiency of the system. The inherent broadcasting nature of wireless mediums helps us to adopt network coding very easily. At the same time, as it reduces the number of transmissions, it reduces the probability of various losses due to transmission impairments, channel contentions and route failures. This paper discusses various well known network coding schemes.. Network coding is broadly classified into global and local network coding. Network coding is also classified into binary - Xor based and random linear coding based network coding. Further to these, based upon the applications, Network coding schemes are classified for unicast, multicast and broadcast applications. In each of these categories, network coding is explained in the reference of inter-session network coding and intra-session network coding.
\end{abstract}

\section{Keywords}

Network Coding, Random Linear Network Coding, Binary Network Coding, Unicast, Multicast, Broadcast, Inter-Session, Intra-Session, COPE, MORE

\section{INTRODUCTION}

Network coding refers to the coding at a node of a specific network. Coding can be either mathematical, substitution or permutation. The basic idea behind network coding is to reduce the number of transmissions. In the earlier days of wireless networking, network suffers from various transmission issues like loss of signal called attenuation, change in signal shapes called distortion, modification of signal called noise. At the same time, when multiple nodes want to communicate all together, they content for the channel which either create problem like hidden or exposed stations or in critical cases collision. Several approaches have been proposed to make the networks robust against such issues. But the ultimate solution is to transmit more in less number of packets. At application layer, data compression can be done. At physical layer, multiplexing our signals at various unique frequencies can be done to generate a composite signal. But what about the middle three layers? Network Coding targets the reduction in number of transmissions corresponding to each of the intermediate layers which are data link layer, network layer and physical layer. This paper discussed network coding at various layers of TCP/IP protocol suite [1].

Lets understand basic need of network coding with a simple example shown in figure 1 which shows a network scenario without network coding.

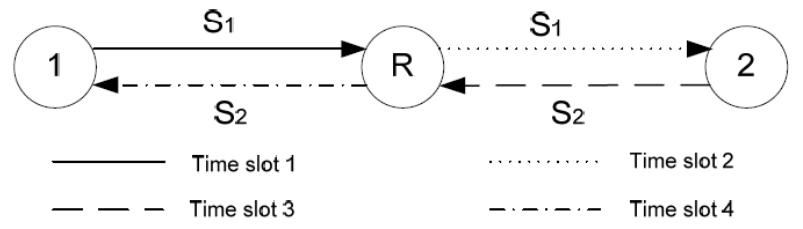

Figure 1. Network without Network Coding

Here node 1 and node 2 want to exchange message S1 and message S2 respectively. The chain topology is formed using intermediate relay node $\mathrm{R}$. in $1^{\text {st }}$ time slot, node 1 sends $\mathrm{S} 1$ to node $\mathrm{R}$. in $2^{\text {nd }}$ time slot, node R sends $\mathrm{S} 1$ to node 2 . In $3^{\text {rd }}$ time slot, node 2 sends $\mathrm{S} 2$ to node $\mathrm{R}$ and in $4^{\text {th }}$ time slot, node $\mathrm{R}$ sends S2 to node 1 . In this way, in 4 transmissions both the messages are exchanged. Our goal is to reduce this requirement to the best by reducing number of transmissions [1].The following sections show various ways of implementing network coding at various layers.

\section{NETWORK CODING AT PHYSICAL LAYER}

PNC - Physical layer network coding is developed for wireless networks. PNC performs network coding when electromagnetic signals are superimposed on each other to form a composite signal which can be transmitted as a single signal. In wireless communication, there are various transmission impairments like attenuation, distortion, noise, cross talk occur which may change the information being transmitted. Interference is considered as a destructive element as it changes the signal and so destructs the information insides. When multiple transmitters transmit signals to various receivers, A receiver may receive signal from its own transmitter as well as from few of the others. The signals from other transmitters are often considered as interferences. When collision occurs, none of the packets can be received correctly due to corruption in both the signals. The same interference can be well treated to take advantage of combining more than one signal. The network coding at physical layer is based on showing positive attitude towards the composite signals. The only difference here is the composite signal is made here is intentional and is reversible[2].

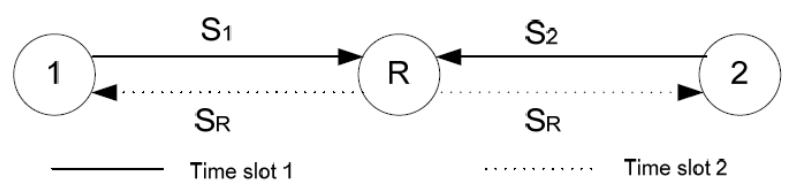

Figure 2. Physical Layer Network Coding 
Figure 2 shows network coding at physical layer. Here node 1 and node 2 transmit S1 and S2 simultaneously during $1^{\text {st }}$ time slot. The relay node receives both and multiplexes both the signals to form a composite signal $\mathrm{SR}$ which it sends to both node 1 and node 2 by broadcasting in $2^{\text {nd }}$ time slot. Node 1 and node 2 will receive $S R$ and will able to decode S2 and S1 message respectively as they already have $\mathrm{S} 1$ and $\mathrm{S} 2$ message respectively. So here a total of 2 transmissions are needed. The mapping of both the signals $\mathrm{S} 1$ and $\mathrm{S} 2$ at relay node $\mathrm{R}$ is called PNC mapping. Various methods like QPSK Quadrature Phase Shift Keying are used for the same. Physical layer network coding is best but comparatively costly and complex. So there are other non physical layer network coding schemes which are becoming more popular. These methods are also called straightforward because of its implementation simplicity[2].

Node $\mathrm{R}$ broadcasts $\mathrm{SR}=\mathrm{S} 1 \oplus \mathrm{S} 2$ where $\bigoplus$ is $\mathrm{XOR}$

Node 1 has $\mathrm{S} 1$ and so it decodes

$\mathrm{S} 2=\mathrm{S} 1 \oplus \mathrm{SR}=\mathrm{S} 1 \oplus(\mathrm{S} 1 \oplus \mathrm{S} 2)$

Node 2 has S2 and so it decodes

$\mathrm{S} 1=\mathrm{S} 2 \bigoplus \mathrm{SR}=\mathrm{S} 2 \bigoplus(\mathrm{S} 1 \oplus \mathrm{S} 2)$

Let's say S1 = 10110 and S2 = 11011

$\mathrm{SR}=\mathrm{S} 1 \bigoplus \mathrm{S} 2=10110 \oplus 11011=01101$

$\mathrm{S} 2$ can be retrieved at Node 1

$\mathrm{S} 2=\mathrm{S} 1 \oplus \mathrm{SR}=10110 \oplus 01101=11011$

$\mathrm{S} 1$ can be retrieved at Node 2

$\mathrm{S} 1=\mathrm{S} 2 \bigoplus \mathrm{SR}=11011 \oplus 01101=10110$

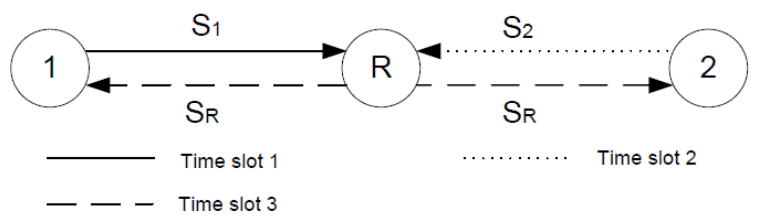

Figure 3. Straight forward Network Coding

Figure 3 shows an example of straight forward packet based network coding. In $1^{\text {st }}$ time slot, node 1 sends $S 1$ to $R$. in $2^{\text {nd }}$ time slot node 2 sends S2 to R. R performs network coding SR which it broadcasts to node 1 and node 2 in $3^{\text {rd }}$ time slot. Subsequently both node 1 and node 2 will retrieve S2 and S1 respectively. Here the number of transmissions is reduced to $3[2][3]$.

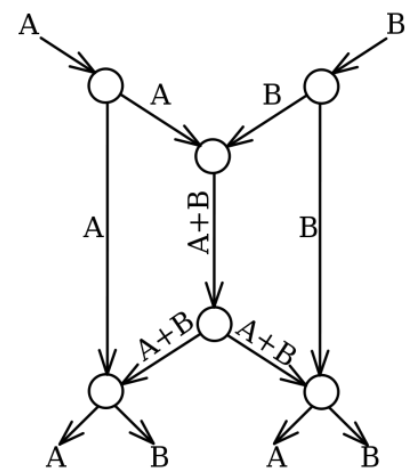

Figure 4. Network Coding in Butterfly Network
Figure 4 shows an example of straight forward packet based network coding for a butterfly network. The various transmissions are shown based on the transmission range and carrier sensing ranges. Every arc is able to carry one packet in a specific direction. The straightforward network coding is also known as linear network coding. Figure 5 shows a simplified way of describing network coding at a node.

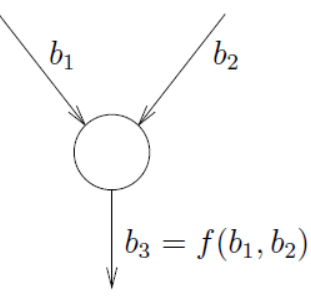

Figure 5. Network Coding

\section{LINEAR NETWORK CODING}

Figure 1, 2 and 3 are examples of linear network coding. These coding schemes are called linear because they use a linear function like Xor operation to code the packets. Linear network coding can be classified with following categories [3].
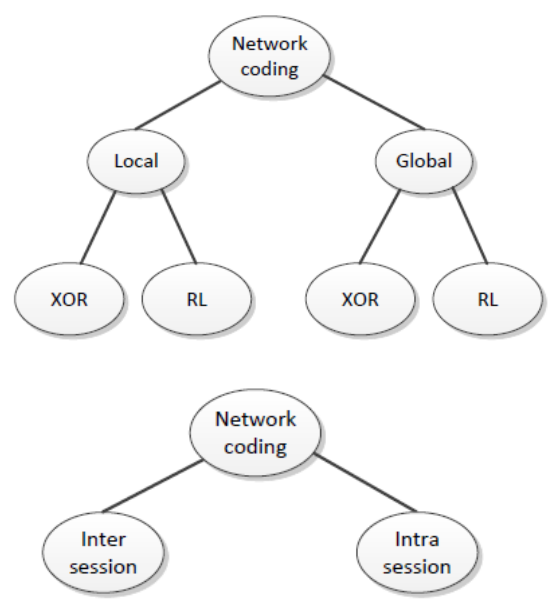

Figure 6. Linear Network Coding Classification

Linear coding with XOR operation can be seen in Figure 3 and Figure 4 which is called binary linear network coding. One another category is Random Linear Coding. One another classification based on the source of packets being coded inter session and intra session network coding.

Network coding can be local or global. In local network coding, the intermediate nodes can decode the coded packets and can code them again. In global network coding, intermediate nodes cannot decode packets and so they don't code them again. Local network coding is based on XOR while global network coding is based on random network coding [4].

\subsection{Inter Session Network Coding}

Lets assume that in figure 3 , node 1 and node 2 are not directly in range of the communication. And so they must have to communicate via a relay node $\mathrm{R}$. here as explained, using XOR function at relay node $\mathrm{R}$, node 1 and node 2 can receive each other's message in less number of transmissions. At the same time, inter session network coding solves the bottleneck problem too. A network coding where more than packets of different sources are coded together is called inter 
session network coding. Inter session network coding's primary goal is to provide higher throughput with less interference [5].

\subsection{Intra Session Network Coding}

In a single session, to provide the reliability, the sent packets must be acknowledged once properly received by sending feedback messages. Such feedback messages are sent as separate packets which consume bandwidth. The more consumed bandwidth the more chance of interference arise and lesser throughputs can be achieved. Network coding can be used to code data packet and feedback together. This method is sometimes called network coding with piggybacking concept which best suits for bidirectional communication. At the same time, over unreliable link, a source can send coded form of more than one packet to ensure reliable delivery[5].

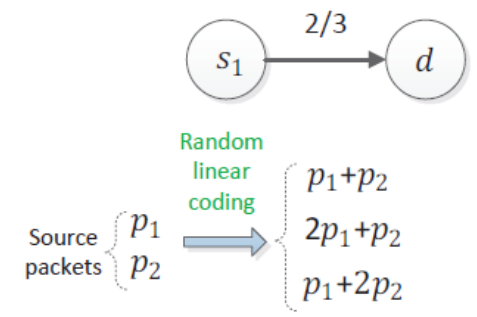

Figure 6. Intra Session Network Coding for reliability

Figure 6 shows an example where a link from source node S1 to destination node $\mathrm{d}$ has reliability of $2 / 3$. It means on average 2 out of 3 packets can be received successfully. For example, node S1 wants to send two packets P1 and P2. Without network coding, because of the unreliable link, node $\mathrm{S} 1$ needs to either send all the two messages twice in case of no feedback based concept. Here it will be 4 . In case of feedback based concept it has to retransmit lost packets on receive of negative acknowledgement. The better solution is to use network coding where three coded packets are sent[5].

Let's solve this example with the help of random linear network coding. Here the source has a set of packets .Let's say a source wants to send $K$ packets $\left\{\mathrm{P}_{1}, \mathrm{P}_{2}, \ldots \mathrm{P}_{\mathrm{k}}\right\}$. for each packet, source selects a coefficient from a finite field randomly and uniformly which is $\left\{\alpha_{1}, \alpha_{2}, \ldots \alpha_{k}\right\}$. the source forms the coded packet of the form,

$$
\sum_{i=1}^{k} \alpha \mathrm{i} * \mathrm{Pi}
$$

The set of coefficients form the encoded vector $\alpha$. It is sent along with the packet as a part of the header so the destination can use it for the decoding purpose. Thus network coding forms a sequence of linear equations which can be solved using Gauss-Jordan elimination method to find the original packets[5].

Packet_1:- P1 + P2

Coefficient Vector V1 $=[1,1]$

Packet_2:- $2 \mathrm{P} 1+\mathrm{P} 2$

Coefficient Vector V2 $=[2,1]$

Packet_3:- $\mathrm{P} 1+2 \mathrm{P} 2$

Coefficient Vector V3 $=[1,2]$

As per the unreliability, if $d$ receives Packet_1 and packet_2,

$\mathrm{P} 1=$ Packet $\_2-$ Packet_1 $=2 \mathrm{P} 1+\mathrm{P} 2-\mathrm{P} 1-\mathrm{P} 2$

$\mathrm{P} 2=$ Packet $\_1-\mathrm{P} 1=\mathrm{P} 1+\mathrm{P} 2-\mathrm{P} 1$

As per the unreliability, if d receives Packet_1 and packet_3,

$\mathrm{P} 2=$ Packet_3 - Packet_1 $=\mathrm{P} 1+2 \mathrm{P} 2-\mathrm{P} 1-\mathrm{P} 2$

$\mathrm{P} 1=$ Packet $\_1-\mathrm{P}_{2}=\mathrm{P} 1+\mathrm{P}_{2}-\mathrm{P} 2$

As per the unreliability, if d receives Packet_2 and packet_3,

Now Gauss-Jordan elimination method is needed.

Lets take a simple example where $\mathrm{P} 1=2$ and $\mathrm{P} 2=3$.

As per the Packet_2 and Packet_3 with Coefficient V2 and V3 respectively,

Packet_ $2=(2 * 2)+3=7 \quad \rightarrow 2 \mathrm{P} 1+\mathrm{P} 2=7$

Packet_3 $=2+(2 * 3)=8 \quad \rightarrow \mathrm{P} 1+2 \mathrm{P} 2=8$

(1) The matrix can be represented.

(2) Find the pivot in the 1st column and swap the 2nd and the 1st rows.

(3) Eliminate the $1^{\text {st }}$ column.

(4) Make the pivot in the 2nd column by dividing the 2nd row by -3

(5) Eliminate the $2^{\text {nd }}$ column

\begin{tabular}{|c|c|c|c|}
\hline & $\mathrm{C} 1$ & $\mathrm{C} 2$ & $\mathrm{~V}$ \\
\hline $\mathrm{P} 1$ & 2 & 1 & 7 \\
\hline $\mathrm{P} 2$ & 1 & 2 & 8 \\
\hline
\end{tabular}

(1)

\begin{tabular}{|c|c|c|c|}
\hline & $\mathrm{C} 1$ & $\mathrm{C} 2$ & $\mathrm{~V}$ \\
\hline $\mathrm{P} 1$ & 1 & 2 & 8 \\
\hline $\mathrm{P} 2$ & 0 & -3 & -9 \\
\hline
\end{tabular}

(3)

\begin{tabular}{|c|c|c|c|}
\hline & $\mathrm{C} 1$ & $\mathrm{C} 2$ & $\mathrm{~V}$ \\
\hline $\mathrm{P} 1$ & 1 & 0 & 2 \\
\hline $\mathrm{P} 2$ & 0 & 1 & 3 \\
\hline \multicolumn{4}{|c|}{$(5)$}
\end{tabular}

Which is the decoding. $\mathrm{P} 1=2$ and $\mathrm{P} 2=3$.

This method has two major disadvantages.

1. Decoding process is time consuming because of the complexity of Gauss-Jordan elimination method.

2. It increases transmission overhead because of sending coefficients vector for the decoding purpose.

The network coding techniques discussed in this topic are related to the broadcast / multicast communication.

\section{UNICAST NETWORK CODING 4.1 Inter Session Network Coding}

COPE is the first unicast network coding technique which is implemented in a real scenario. At the same time, from the simulation point of view, it is simulated in NS simulator. COPE shows a significant amount of performance improvement over multihop networks. Xor in the air is the main tagline of COPE and so it is categorized under XOR based linear network coding. The key concept is same. To reduce number of transmissions, to save overall communication time and to reduce the need of the resources, 
an intermediate node can forward as well as code multiple packets which will be subsequently forwarded or coded by next hopes. COPE resides inside a thin layer between IP and MAC. COPE keeps looking for opportunities to perform network coding. COPE is based on three techniques[6].

\section{Opportunistic Listening}

Opportunistic listening identifies and increases opportunities of implementing network coding. All nodes keep listening everything and so receive all packets. Every node tries to decode all the packets doesn't matter whether they are actually intended to them or not. COPE makes nodes to work on promiscuous mode. Every node stores the overhead packets for a particular amount of time mostly of 0.5 Seconds. By broadcasting reception reports, a node informs all the neighboring nodes which packets it has decoded and stored[6].

\section{Opportunistic Coding}

Opportunistic coding is used to find which packets to code. Network coding is performed across the neighboring nodes. A set of nodes mostly have redundant packets. COPE defines a set of rules to perform selection of packets to perform network coding. Based on these rules, every node performs network coding of selected packets available in its local buffer. Generally a node finds multiple ways of doing network coding, but it should select the way which maximizes the number of native - unencoded packets delivery through a single transmission. At the same time it must ensure that each intended next node has sufficient packets to decode the packets being sent[6].

Figure 7 shows one example of opportunistic coding. Every node has certain packets in their local buffers. A node broadcasts reception reports to all the neighbors to let them know what packets it has. So it can be assumed that at present every node knows which packets are available at every other node. At the same time every node has a queue of packets waiting for the transmission. Suppose Node B has for packets in its output queue - P1, P2, P3 and P4. In opportunistic coding, node $\mathrm{B}$ tries to find the best encoding scheme which transmits more packets in less number of transmissions at the same time, which makes decoding possible at maximum number of neighboring nodes[6].

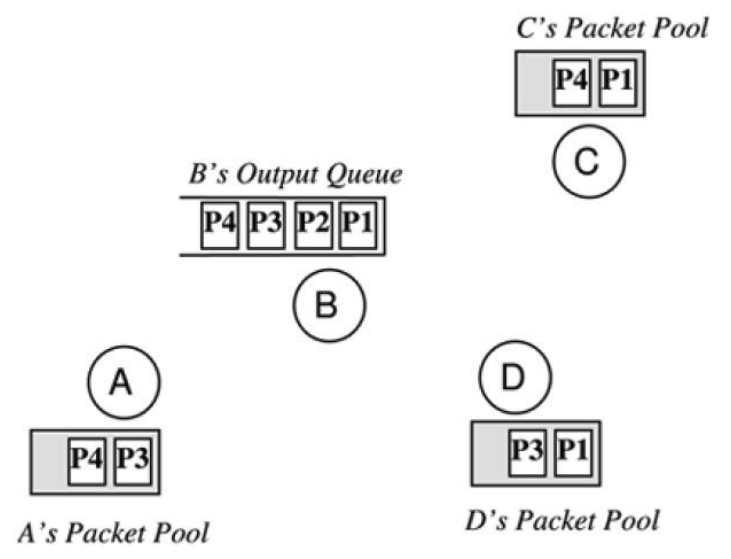

Figure 7. COPE Example
Lets discuss three options. If Node $\mathrm{B}$ codes $\mathrm{P} 1$ and $\mathrm{P} 2$ as a packet $\mathrm{P} 1 \oplus \mathrm{P} 2$, on broadcast, Node $\mathrm{C}$ will be able to decode P2 because it has P1. Node D will be able to decode P2 because it has P1 but Node A will not able to decode anything because it has neither P1 nor P2[6].

The same way, if Node B codes P1 and P3 then it forms packet $\mathrm{P} 1 \bigoplus \mathrm{P} 3$, on broadcast, Node $\mathrm{A}$ and Node $\mathrm{C}$ will be able to decode $\mathrm{P} 1$ and $\mathrm{P} 3$ respectively. But there will be no benefit at Node D because it already has P1 and P3[6].

The best option that Node B should select is to form a packet as P1 $\bigoplus \mathrm{P} 3 \oplus \mathrm{P} 4$, on broadcast, Node A will be able to decode $\mathrm{P} 1$, Node $\mathrm{C}$ will be able to decode $\mathrm{P} 3$ and Node $\mathrm{D}$ will be able to decode $\mathrm{P} 4$. So in a single transmission, all the three packets P1, P3 and P4 can be avail to all the nodes[6].

In the next transmission, Node B simply broadcasts Packet P2 and all other nodes receive it. Here no need of network coding as only one packet is remaining[6].

This method is based on unicast because individual packets are coming from unicast flow. In opportunistic networking, there are no strict routing rules associated with a multihop network and so whoever gets a chance, can forward the packet[6][7].

Mathematically one can define a rule for maximum number of packets a node can encode to maximize the network coding performance as below.

A node having $\mathrm{n}$ packets, $\mathrm{P} 1, \mathrm{P} 2, \ldots$ Pn wants to transfer packets to $n$ next hops, h1, h2,...hn. In this case, a node can XOR the m packets, if each of the next hop has m-1 packets. This defines that each next hop has $\mathrm{m}-1$ packets and so each node will be able to decode $\mathrm{m}^{\text {th }}$ packet[6][7].

\section{Learning Neighboring State}

The basic requirement is that every node knows about what packets other nodes have. After that only, a node can take an optiomal coding decision. Every node broadcasts list of packets it has as a reception reports. But during congestion, such reports may be lost due to collisions. Even during the light traffic, sometimes, reception reports arrive late and meanwhile, a node might have taken decision about which packets to code. In such cases, a node should not completely dependent on reception reports. A node should intelligently guess what other nodes must be having. Link state routing protocols are based on calculating delivery probabilities of every link to compute shortest and reliable path. COPE uses this information to predicate whether a node has a specific packet or not. Delivery rates are calculated and broadcasted frequently to all the nodes[7].

Lets take an another example which is based on taking coding decision based on delivery rates rather than of reception report[7]. 


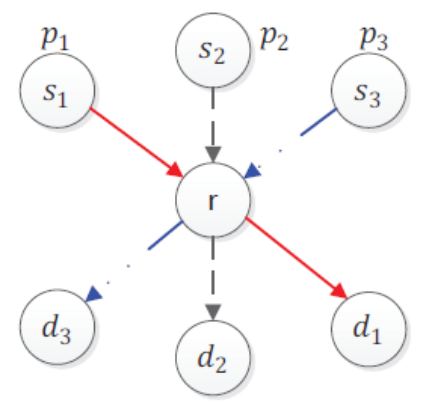

Figure 8. Opportunistic Network Coding Example

Figure 8 shows an example where nodes S1, S2 and S3 have next hops $\mathrm{d} 1, \mathrm{~d} 2$ and $\mathrm{d} 3$ respectively via a relay node $\mathrm{r}$. lets say delivery probability of each of the links is 1 . But overhearing probability of each of the links is 0.8 . in COPE the threshold of overhearing probability is also 0.8 [7].

Once a relay node $\mathrm{r}$ receives $\mathrm{P} 1, \mathrm{P} 2$ and $\mathrm{P} 3$, it has to take decision of encoding. Relay node $r$ selects $\mathrm{P} 1$ with the probability 1 . Next time it selects $\mathrm{P} 2$, forming P1 $\bigoplus \mathrm{P} 2$ with overhearing probability $1 * 0.8=0.8$ which is equal to the COPE threshold. Next time it select P3, forming P1 $\bigoplus$ P2 $\bigoplus$ P3 with overhearing probability $1 * 0.8 * 0.8=0.64$ which is less than COPE threshold. So a relay node $\mathrm{r}$ can code maximum two packets. $\mathrm{P} 1 \oplus \mathrm{P} 2$ or $\mathrm{P} 1 \oplus \mathrm{P} 3$ or $\mathrm{P} 2 \bigoplus \mathrm{P} 3$ and then send the third packet, $\mathrm{P} 3$ or $\mathrm{P} 2$ or $\mathrm{P} 1$ as a second transmission. Usually COPE selects packets in FIFO manner[8].

\subsection{Intra Session Network Coding}

COPE is used with inter session network coding and so based on XOR based network coding. As discussed in 3.2, intra session network coding resides inside a source. Here MORE is one of the most popular intra session network coding method based on random linear network coding for unicast and multicast applications[9][10].

In traditional routing, path from source to destination is predetermined. In opportunistic routing, any node which can overhear the transmission and which is presently more closer to the destination can forward the packet. the issue here is that more than one nodes may overhear the transmissions and may forward the same packets if they feel they are closer to the destination. MORE tries to resolve this issue where unnecessary opportunistic transmissions can be avoided [9][10].

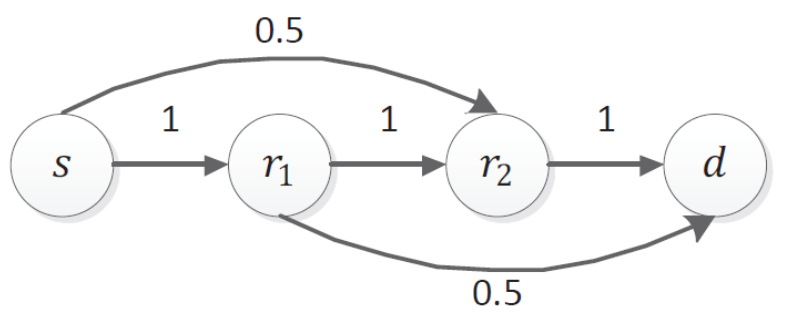

Figure 9. MORE Example

Figure 9 shows a possible multihop scenario where source node s tries to send two packets $\mathrm{P} 1$ and $\mathrm{P} 2$ to destination node $\mathrm{d}$ via two relay nodes $\mathrm{r} 1$ and $\mathrm{r} 2$. In traditional routing, packets follow the path from $\mathrm{s} \rightarrow \mathrm{r} 1 \rightarrow \mathrm{r} 2 \rightarrow \mathrm{d}$. lets think from the carrier sense and transmission range point of view. Node $s$ sends P1 and P2 to relay $\mathrm{r} 1$ which $\mathrm{r} 1$ has to forward to $\mathrm{r} 2$. But what if $\mathrm{r} 2$ can detect $\mathrm{P} 1$ as an overhear transmission? It receives $\mathrm{P} 1$ and sends directly to $\mathrm{d}$ as it is nearer to $\mathrm{d}$. in such case, $\mathrm{r} 1$ doesn't need to send P1 to $\mathrm{r} 2$ as it already forwarded. Node r1 needs to send P2 only. MORE avoids such unnecessary transmission using random linear network coding [9][10].

According to MORE, r1 neither send P1 separately nor P2 separately. Node $\mathrm{r} 1$ performs random linear network coding to form a packet $\mathrm{C} 1 \mathrm{P} 1+\mathrm{C} 2 \mathrm{P} 2$ and sends to node $\mathrm{r} 2$. Node $\mathrm{r} 2$ can easily decode the missing packet $\mathrm{P} 2$ by subtracting $\mathrm{C} 1 \mathrm{P} 1$ from it. Note that the coefficients are sent together with the packet.

\section{More Coding}

The source nodes breaks the message into set of $\mathrm{k}$ uncoded packets called native packets. The source node performs random linear network coding and sends a coded packet along with the coefficient vector as a part of the header.

$$
\sum_{i=1}^{k} \alpha \mathrm{i} * \mathrm{Pi}
$$

Where $P i$ is $i^{\text {th }}$ packet and $\alpha \mathrm{i}$ is $i^{\text {th }}$ coefficient.

In MORE, the header also stores the list of forwarder nodes. Forwarder list can be calculated by using ETX - Expected number of Transmissions. Every forwarded node which receives this packet first checks, whether the packet has new information? New information refers to the packet which is innovative. A packet is innovative if it is encoding of a set of packets which are linearly independent from the previously coded packets. If a node finds an innovative packet, it decodes it and tries to broadcast it again in case of local network coding. In case of global network coding, it broadcasts as it is. The destination will be able to decode all the packets once it receives all the $\mathrm{k}$ innovative packets [9][10].

\section{MULTICAST NETWORK CODING}

With modification to the unicast network coding schemes, multicast network coding schemes can be formed. At present there are no standard inter session multicast network coding schemes for wireless multihop networks are proposed. As this paper discusses network coding with the reference of wireless multihop networks, inter session multicast network coding for wired networks is not discussed [11].

\subsection{Intra Session Network Coding}

MORE's unicast approach can be modified to get multicast network coding easily. MORE follows following two conditions. MORE source can not proceed to another batch of packets until all the destinations intended for current batch have not received and have not acknowledged. MORE redefines the forwarder list. MORE source determines a list of forwarder nodes for each of the unicast flow inside the multicast flow. The forwarder list for multicast is a union of the forwarder lists of unicast flows [11].

Multicasting with MORE invites two major issues. Because of too many forwarding nodes, congestion can be an issue. The situation becomes worst as the number of destination increases. Multicasting suffers from crying baby problem. When one receiver has poor reliability in the form of connectivity to the networks, time will be wasted in fulfilling delivery for it. This is because MORE source is not allowed to initiate another batch until the current batch is delivered at all the multicast destinations [11]. 
To resolve crying baby problem, MORE's variant, Pacifier changes the pattern of initiating batches. In MORE, a source doesn't start a new batch until all the destinations acknowledge the current batch. So in a way, it is like a batch processing. Pacifier suggests round robin kind of pattern. A source MORE can start a new batch when one of the receivers (receivers may be intermediate hops) acknowledges receive of the current batch. Subsequently, current batch will be propagated in the network. At the same time next batch will started too [11].
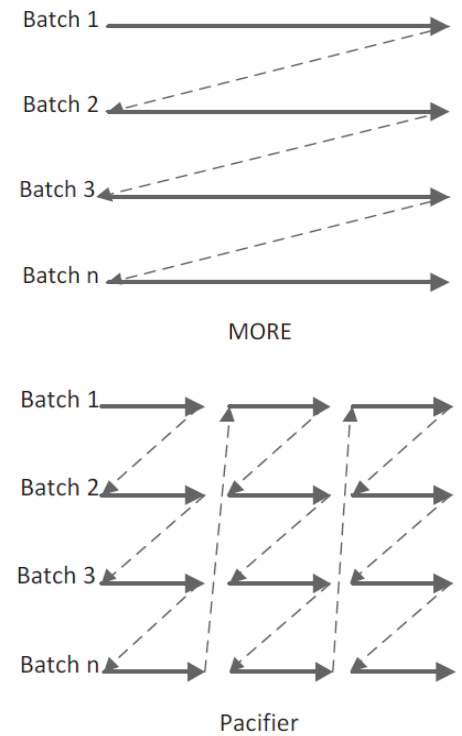

Figure 10. MORE Vs Pacifier Pattern

\section{BROADCAST NETWORK CODING 6.1 Inter Session Network Coding}

A special broadcast problem is all-to-all broadcast, where every node wants to broadcast its packets to all the other nodes. Here the network coding could be applied to reduce the number of transmissions significantly and to increase overall performance too [12]

CODEB method selects a set of relay nodes from all who want to broadcast. Each relay node covers two hop neighbors atleast. Every relay node codes the packets with a combination which can be decoded by its neighbors. The problem itself is NP-Hard and so it is better be solved by greedy approach [12].

One another approach suggests division of omnidirectional geographical area with all the nodes into a set of sectors with directional antennas corresponding to the relay nodes. The purpose here is to define sectors - each of specific number of nodes. Parallel directional transmissions can be done of coded packets. The only problem with this method is the links must be perfect. The process can be visualized with a 3-D hypercube topology as shown in Figure 11 - One to All broadcast and Figure 12 - All to All Broadcast [12].

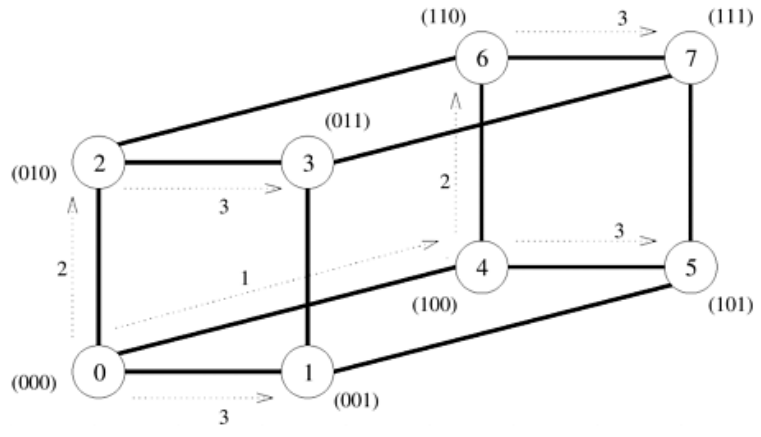

Figure 11. One-to-All Broadcast

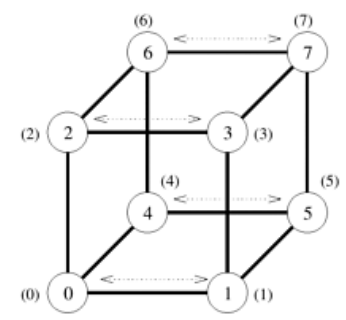

(a) Initial distribution of messages

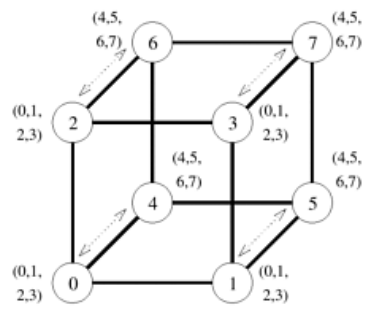

(c) Distribution before the third step

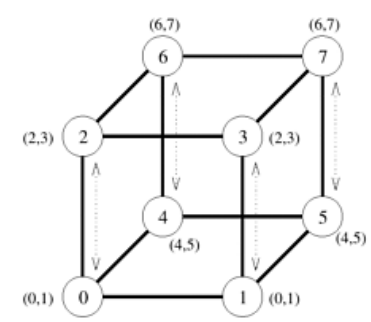

(b) Distribution before the second step

\section{Figure 12. All-to-All Broadcast}

\subsection{Intra Session Network Coding}

NAK- Negative Acknowledgement based network coding schemes are becoming popular for the broadcasting purpose. On loss, source receives NAK message with the information about the lost packets. At the time source receives a NAK message, it doesn't retransmit the lost packets immediately but it keeps the list of lost packets corresponding to each of the possible receivers. Source performs retransmission at regular interval by initiating a retransmission phase. Source codes the lost packets at best and keep retransmitting until all are not acknowledged. For the simplicity of the discussion, lets take an example shown in figure 13 [12].

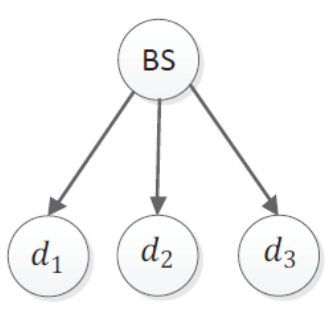

Figure 13. Broadcasting with losses

Let's say BS broadcasts packet P1, P2, P3,P4, P5. Node d1 missed packets $\mathrm{P} 1, \mathrm{P} 2$ and $\mathrm{P} 3$. Node $\mathrm{d} 2$ missed packets $\mathrm{P} 1$ and P4. Node d3 missed packet P5. As node d1 has lost maximum packets, algorithm keeps each of the lost packets by $\mathrm{d} 1$ in separate groups. Let $\mathrm{S} 1=\{\mathrm{P} 1\}, \mathrm{S} 2=\{\mathrm{P} 2\}, \mathrm{S} 3=\{\mathrm{P} 3\}$. 
Packet $\mathrm{P} 4$ can be added into S2 or S3. P4 cannot be added to $\mathrm{S} 1$ because at node $\mathrm{d} 2, \mathrm{P} 1$ and $\mathrm{P} 4$ both are missed. Let's add P4 into S2. Packet P5 can be added to any of the sets as it is missed at only one node d3. Let's add it in S1. So final coding sets are $\mathrm{S} 1=\{\mathrm{P} 1, \mathrm{P} 5\}, \mathrm{S} 2=\{\mathrm{P} 2, \mathrm{P} 4\}, \mathrm{S} 3=\{\mathrm{P} 3\}$.

\section{CONCLUSION}

This paper started with the introduction to the network coding. Network coding at physical layer and above the physical layer too are discussed. Network coding is broadly classified into global and local network coding. Network coding is also classified into binary - Xor based and random linear coding based network coding. Further to these, based upon the applications, we classified network coding schemes for unicast, multicast and broadcast applications. In each of these categories, network coding is further explained in the reference of inter-session network coding and intra-session network coding. There is a lot of scope towards improving network coding schemes. One such area is using prediction based algorithm to find when to use network coding and when not to. A special area of network coding with opportunistic routing is becoming more and more popular as opportunistic networks are the most efficient way of wireless networking. Network coding can also be introduced at application layer for some applications like web browsers etc. When a client has opened multiple sessions through multiple tables of a single browser, corresponding to a specific server, network coding can be applied over them. There is also a way of introducing network coding to code more than one packets belonging to more than one communication flow. Following table compares various network coding schemes.

\begin{tabular}{|c|c|c|c|c|c|}
\hline Scheme & Methodology & Application & Type & Category & Links \\
\hline COPE & Inter-session & Unicast & Xor & Local & Lossy \\
\hline MORE & Intra-session & unicast & RL & Global & Lossy \\
\hline MORE & Intra-session & Multicast & RL & Global & Lossy \\
\hline Pacifier & Intra-session & Multicast & RL & Global & Lossy \\
\hline CODEB & Inter-session & Broascast & Xor & Local & Perfect \\
\hline Dir. Ant. & Inter-session & Broascast & Xor & Local & Perfect \\
\hline NAK & Intra-session & Broascast & RL & Global & Lossy \\
\hline
\end{tabular}

\section{REFERENCES}

[1] Christina Fragouli, Emina Soljanin, "Network Coding Applications",Foundations and Trends in Networking

[2] Christina Fragouli, Jean-Yves Le Boudec, "Network Coding: An Instant Primer" ACM SIGCOMM Computer Communication Review, Volume 36, January 2006.

[3] Hao Kun, Jin Zhigang, Wang Ying, "Partial Network Coding for Wireless Opportunistic Routing", IET Conference Publications 2009.

[4] Shengli Fu, Kejie Lu, Yi Qian, and Murali Varanasi, "Cooperative Network Coding for Wireless Ad-Hoc Networks", IEEE GLOBECOM 2007 proceedings.

[5] Wei Chen, Khaled B. Letaief, and Zhigang Cao, "Opportunistic Network Coding for Wireless Networks", ICC 2007 proceedings [7] Lu Lu, Ming Xiao, Mikael Skoglund, Lars Rasmussen, Gang Wu, Shaoqian Li, "Efficient Network Coding for Wireless Broadcasting", WCNC 2010 proceedings

[6] Shuo-Yen Robert Li, Raymond W. Yeung , "Linear Network Coding", IEEE Transactions on Information Theory, VOL. 49, NO. 2, February 2003

[7] Rodica Stoian, Lucian Andrei Perisoara, Radu Stoica, "Random Network Coding for Wireless Ad-Hoc Networks", Wireless Communications and Networking Conference WCNC IEE 2010

[8] Tracey Ho, Muriel M'edard, Ralf Koetter, David R. Karger, Michelle Effros, Jun Shi and Ben Leong, "A random linear network coding approach to Multicast", IEEE Trans. Information Theory, Oct 2006

[9] Philip Chou, Yunnan Wu, Kamal Jain, "Practical Network Coding", in Proc. Allerton Conf. Commun., 2003

[10] Mohammed Halloush, Hayder Radha, "Network Coding with Multigeneration Mixing", ICC 2008 proceedings

[11] Mohammed Halloush, Hayder Radha, "A Case Study of: Sender Transmission Reliability and Complexity Using Network Coding with Multi-generation Mixing", ICC 2009 proceedings

[12] Mohammed Halloush, Hayder Radha, "Network Coding with Multi- Generation Mixing: Analysis and Applications for Video Communication", ICC 2008 proceedings 